\title{
Gut Microbiota Dysfunction as Reliable Non-invasive Early Diagnostic Biomarkers in the Pathophysiology of Parkinson's Disease: A Critical Review
}

\author{
Arun T Nair, ${ }^{1}$ Vadivelan Ramachandran, ${ }^{1 *}$ Nanjan M Joghee, ${ }^{2}$ Shanish Antony, ${ }^{3}$ and Gopalakrishnan Ramalingam ${ }^{1}$ \\ ${ }^{I}$ Department of Pharmacology, ${ }^{2}$ JSS College of Pharmacy (JSS Academy of Higher Education and Research, Mysuru), Ootacamund, Tamilnadu, \\ India, and ${ }^{3}$ Department of Pharmacology, Government Medical College, Kottayam, Kerala, India
}

Recent investigations suggest that gut microbiota affects the brain activity through the microbiota-gut-brain axis under both physiological and pathological disease conditions like Parkinson's disease. Further dopamine synthesis in the brain is induced by dopamine producing enzymes that are controlled by gut microbiota via the microbiota-gut-brain axis. Also alpha synuclein deposition and the associated neurodegeneration in the enteric nervous system that increase intestinal permeability, oxidative stress, and local inflammation, accounts for constipation in Parkinson's disease patients. The trigger that causes blood brain barrier leakage, immune cell activation and inflammation, and ultimately neuroinflammation in the central nervous system is believed to be due to the chronic low-grade inflammation in the gut. The non-motor symptoms that appear years before motor symptoms could be reliable early biomarkers, if they could be correlated with the established and reliable neuroimaging techniques or behavioral indices. The future directions should therefore, focus on the exploration of newer investigational techniques to identify these reliable early biomarkers and define the specific gut microbes that contribute to the development of Parkinson's disease. This ultimately should pave the way to safer and novel therapeutic approaches that avoid the complications of the drugs delivered today to the brain of Parkinson's disease patients.

(J Neurogastroenterol Motil 2018;24:30-42)

Key Words

Biomarkers; Constipation; Gastrointestinal microbiome; Humans alpha synuclein; Parkinson disease

\section{Introduction}

Parkinson's disease (PD) is considered today as the most common movement disorder, which affects 1-2 per 1000 of the population worldwide. ${ }^{1}$ It has been estimated that 7-10 million people in the world have PD. ${ }^{2}$ This is expected to double in the next 2 decades in proportion with the increase in the aging population. ${ }^{3}$ Complexity of the etiology of this disease along with clinical challenges, including difficulty to make definite early stage diagnosis and management of symptoms in the later stage, make treatment of PD difficult. ${ }^{4}$ Even though motor symptoms are still considered to

Received: September 1, 2017 Revised: November 8, 2017 Accepted: November 20, 2017

(.) This is an Open Access article distributed under the terms of the Creative Commons Attribution Non-Commercial License (http://creativecommons. org/licenses/by-nc/4.0) which permits unrestricted non-commercial use, distribution, and reproduction in any medium, provided the original work is properly cited.

${ }^{*}$ Correspondence: Vadivelan Ramachandran, PhD

Department of Pharmacology, JSS College of Pharmacy ((JSS Academy of Higher Education and Research, Mysuru), Ootacamund, Tamilnadu 643001, India

Tel: +91-9047539532, Fax: +91-423-2442937,E-mail: vadivelanr@jssuni.edu.in 
be the hallmarks of PD, early non-motor symptoms (NMS) have been given considerable attention during the last 2 decades. ${ }^{5}$ These NMS could definitely be considered as either an indicator of risk factors that lead to PD or an early biomarker that stand as an objective measure to identify PD pathogenesis. ${ }^{3}$ In the present review, we explore the diagnostic potential of NMS associated with gut microbiota dysfunction. These early symptoms that are believed to appear during the preclinical stage of the disease have to be correlated with the established investigational techniques to discover reliable early non-invasive PD biomarkers. It is hoped that the present review will lead to further intensive research leading to successful detection of early stage biomarkers for the safe and effective treatment of PD.

\section{Parkinson's Disease}

Worldwide non-communicable diseases play a major role for mortality compared to communicable diseases. ${ }^{6}$ Neurodegenerative diseases, the main culprit of disability, are increasing globally at a fast pace. ${ }^{7-10} \mathrm{PD}$ is a multifactorial neurodegenerative disorder characterized by motor symptoms like dyskinesia, muscular rigidity, tremor, slowness of movement, and gait disorder. Apart from these motor symptoms, NMS like depression, dementia, autonomic and sensory dysfunction also accompany PD. In addition to the widely known dopaminergic loss in the substantia nigra pars compacta, $\mathrm{PD}$ is also characterized by synucleinopathy, the accumulation of insoluble polymers of denatured alpha-synuclein ( $\alpha$-synuclein) along with toxic parkin substrates called lewy bodies in the neurons. Lewy bodies are considered to be responsible for neurodegeneration and neuronal death. ${ }^{11,12}$

\section{Conventional Diagnosis, Treatments, and Their Shortcomings}

The conventional diagnosis of PD relies on the patient's clinical symptoms and neurological examination. Specialized brain scanning techniques that measure dopamine system and brain metabolism are among the best options available today to diagnose PD. ${ }^{13}$ The currently employed treatment for PD using drugs like levodopa, dopamine agonists, monoamine oxidase (MAO) B inhibitors, anticholinergic agents, amantadine, and catechol-O-methyl transferase inhibitors have limitations with regard to both efficacy and safety. ${ }^{14}$ Prolonged use of levodopa is associated with motor fluctuations and dyskinesia while dopamine agonists are associated with an increased risk of impulse control disorders. The MAO B inhibitors have only a modest symptomatic benefit as monotherapy. The use of anti- cholinergic drugs is limited due to their side effects. The use for older or demented individuals and those without tremor is strongly discouraged. Amantadine is a relatively weak antiparkinsonian drug and its toxic side effects are more likely in older patients. The main drawback of the conventional treatment is that it cannot stop the progress of disease or reverse the symptoms. Furthermore, the conventional diagnosis of PD relies mainly on the motor symptoms of the patient. By the time motor symptoms emerge, however, a significant amount of neurological damage and destruction of structural changes might have already taken place. In addition, the neuroimaging tests employed are quite expensive and can be performed only in specialized imaging centers. What is more, today there is no standard diagnostic test for PD.

\section{Importance of Early Diagnosis Through Biomarkers}

Early diagnosis should enable immediate treatment and help protect the neuronal functions, slow disease progression, improve patient's quality of life and ultimately reduce the overall cost of PD treatment. ${ }^{15}$ Recent investigations that use diverse techniques to recognize NMS that precede years before the motor symptom should help in earlier diagnosis of $\mathrm{PD}$ and determine appropriate treatment strategy and alter the diagnostic concept of PD. ${ }^{16}$ Currently there are no reliable biomarkers available that can predict the onset of $\mathrm{PD}$, diagnosis, progression of the disease, and evaluation of the response to disease modifying treatments (DMT).${ }^{17}$ Development of early diagnostic biomarkers at the very beginning is therefore crucial for therapeutic interventions. Biomarkers for PD are classified generally into three types, namely prodromal, preclinical or premotor stage, risk or susceptibility stage, and motor stage. ${ }^{18,19}$ The prodromal biomarkers which could predict the onset of $\mathrm{PD}$ years in advance are, no doubt, very crucial. These biomarkers can again be classified mainly into 3 types, namely clinical biomarkers, biochemical biomarkers and imaging biomarkers (Table 1). The clinical NMS appear years before the appearance of motor symptoms. ${ }^{17}$ The most common clinical NMS that occur ten years before motor symptoms are constipation, dream enacting behavior, and frequent nightmares (with rapid eye movement, sleep behavior disorder), excessive day time sleepiness, and post prandial fullness. Additionally, the more frequent NMS that are believed to occur 2-10 years before motor symptoms are smell loss and mood disturbances, taste loss, excessive sweating, fatigue, and pain. It is therefore worth considering these clinical NMS for developing early diagnostic biomarkers. 
Table 1. Characteristics of Presymptomatic Stage Biomarkers in Parkinson's Disease (Adapted From Picillo et $\mathrm{al}^{17}$ )

\begin{tabular}{lll}
\hline Presymptomatic biomarker type & \multicolumn{1}{c}{ Subtype } & \multicolumn{1}{c}{ Identifiable characteristics. } \\
\hline Clinical biomarkers & $\begin{array}{c}\text { NMS (appear10 years before motor } \\
\text { symptoms) } \\
\text { NMS (appear between 2-10 years } \\
\text { before motor symptoms) }\end{array}$ & $\begin{array}{c}\text { Constipation, dream enacting behavior, frequent nightmares, excessive } \\
\text { daytime sleepiness, post prandial fullness, tremor } \\
\text { Smell loss, mood disturbances, excessive sweating, fatigue, pain, con- } \\
\text { stipation, hypotension, urinary dysfunction, erectile dysfunction }\end{array}$ \\
& Metabolic factors & Cholesterol level \\
Biochemical biomarkers & Neurotrophic factors & High IGF-1 \\
& Oxidative stress & Low vitamin D \\
& Nuclear imaging & High uric acid level \\
Tmaging biomarkers & Nigrostriatal degeneration \\
& Magnetic resonance imaging & Substantia nigra hyperechogenicity \\
& Reorganization of corticostriatal circuits in mutations
\end{tabular}

NMS, non-motor symptoms; IGF, immunoglobulin F.

The appearance of NMS during the prodromal stage indicates the widespread neuroanatomical and neurochemical changes which occur throughout PD. It involves not only the dopaminergic nigrostriatal system but also serotonergic, noradrenergic and cholinergic frontal and brain stem regions. ${ }^{20}$ Development of prodromal biomarkers could be achieved by determining the relationship between risk factors, hyperechogenicity of the substantia nigra, and positive family history. ${ }^{21}$ The most promising potential clinical prodromal PD biomarkers include bowel dysfunction, RBD, olfactory deficits, and mood disorders. Identification of the potent clinical premotor biomarkers that could support to confirm the sensitivity and specificity of other early phase biomarkers of PD is therefore very crucial.

\section{Communications Between the Brain and the Gastrointestinal Tract}

The microbiota-gut-brain axis is a bidirectional communication between the central nervous system (CNS) and the gastrointestinal (GI) tract. ${ }^{22,23}$ This axis is responsible for the modulation of digestive process, immune function, perception and emotional response to visceral stimuli under physiological conditions. ${ }^{24}$ Various diseases like anxiety and depressive disorders, liver diseases, gut inflammation, disorders of GI motility, and chronic abdominal pain syndrome, etc, are believed to occur due to dysregulation in the microbiota-gut-brain axis. ${ }^{22,25-31}$ This axis incorporates the CNS, autonomic nervous system, and enteric nervous system in addition to the neuroendocrine and the neuroimmune systems. ${ }^{29}$ Afferent fibers from the gut to cortical centers, cerebral, anterior and posterior cingulate, insular and amygdala cortices, as well as effector fibers projecting to the smooth muscles of the gut, stand as the major route for bidirectional communication along the axis. ${ }^{32}$ This network plays a major role and impacts both GI and brain function. ${ }^{31,33}$ Further, dopamine synthesis in the brain is induced by dopamine producing enzymes whose synthesis or inhibition is controlled by gut microbiota via the microbiota-gut-brain axis. ${ }^{34}$

\section{Gut Microbiome Imbalance Affects Brain Through Microbiota-Gut-Brain Axis}

The human microbiome is believed to constitute 100 trillion microorganisms ${ }^{7}$ and the vast majority live in the gut microbiota. ${ }^{35}$ The main chunk of these microbiomes is inherited maternally through the microbiota enriched vaginal fluid of the mother at the time of birth and becomes a vital part of our immunity. ${ }^{36-41}$ These microbiomes act as a barrier between humans and the external environment and plays a major role in the protection against various external hazards. ${ }^{62-46}$ Although the microbiome is inherited from the mother, eventually the microbiome changes to one's own unique signature. ${ }^{39,47,48}$ The gut microbiota changes constantly according to our food habits and the environment. ${ }^{49,50}$ The stool and the intestinal mucosal microbiome compositions may differ from each other and between different individuals. Recent investigations suggest that gut microbiota affects the brain activity through microbiotagut-brain axis under both physiological and pathological disease conditions like $\mathrm{PD}^{25,51-53}$ through a prion like transmission via the molecular mimicry pathway. ${ }^{54}$ 


\section{Recent Perspectives of Parkinson's Disease and Gastrointestinal Tract Dysfunction}

\section{Enteric $\alpha$-Synuclein Triggers Early Gut Symptoms}

Recent reports claim that the origin of $\mathrm{PD}$ does not take place in the substantia nigra alone and its pathology involves extranigral regions as well. Initially lewy bodies begin to appear in the nondopaminergic neurons outside the basal ganglia in the olfactory pathways, namely the gigantocellular reticular nucleus, caudal raphe nuclei, coeruleus-subcoeruleus complex and glosso-pharyngeal vagal complex. ${ }^{55} \mathrm{~A}$ recent perspective is that the onus of $\mathrm{PD}$ is shared by the extra neurological region along with the brain. Comorbid GI dysfunctions characterized by constipation accompany almost $80 \%$ of the PD patients. ${ }^{56}$ Also $\alpha$-synuclein deposition and the associated neurodegeneration in the enteric nervous system, characterized by increased intestinal permeability, oxidative stress and local inflammation, accounts for the constipation in PD patients. ${ }^{57-59}$ These pathophysiological symptoms could be observed in the initial stages of $\mathrm{PD}$, years before the important motor symptoms appear, giving support to the hypothesis that PD pathogenesis might have primary connections with the gut. ${ }^{60-64}$

\section{Gut Dysfunction Impacts Brain}

The clinical manifestations of PD in the gut, their symptoms and etiology are shown in Table 2. The table reveals that the majority of symptoms that are of GI origin appear to be early diagnostic tools in neurodegenerative diseases. It is, therefore, important that these symptoms are investigated in detail to understand the exact pathophysiology of PD. Several studies have been carried out in recent years to understand the role of gut microbiota in PD. The concept of linking PD with the low-grade inflammation of the gut has been around for quite some time. In colonic biopsies of PD patients, increased mRNA expressions of proinflammatory cytokines have been observed compared to normal patients. ${ }^{27}$ It is possible that the trigger that causes blood brain barrier leakage, immune cell activation and inflammation, and ultimately neuroinflammation in

Table 2. Early Clinical Manifestations of Parkinson's Disease in Gut, Symptoms, and Etiology (Adapted From Mukherjee et a ${ }^{65,104}$ )

\begin{tabular}{|c|c|c|}
\hline $\begin{array}{l}\mathrm{PD} \text { associated clinical } \\
\text { manifestation in the gut }\end{array}$ & Symptoms & Etiology and test for evaluation \\
\hline Malnutrition & $\begin{array}{l}\text { Weight alteration, increased disease severity or } \\
\text { duration, depression or anxiety, fatigue, } \\
\text { osteoporosis }\end{array}$ & $\begin{array}{l}\text { Loss of appetite, dysphagia, constipation, early satiety, } \\
\text { increased dose of levodopa, vitamin D deficiency, impaired } \\
\text { insulin signaling, mitochondrial dysfunction }\end{array}$ \\
\hline Oral and dental disorders & $\begin{array}{l}\text { Lesser teeth number, teeth caries, gingival } \\
\text { recession, increased tooth mobility, burning } \\
\text { mouth syndrome, bruxism, temporomandibular } \\
\text { disorder, taste impairment }\end{array}$ & $\begin{array}{l}\text { Low frequency tooth brushing, motor or impairment, } \\
\text { apathy, depression, cognitive impairment }\end{array}$ \\
\hline Sialorrhea & $\begin{array}{l}\text { Drooling, dementia , hallucination, orthostatic } \\
\text { hypotension, silent aspiration, laryngeal } \\
\text { penetration of saliva }\end{array}$ & $\begin{array}{l}\text { Dysphagia with less efficient swallowing, abnormal head } \\
\text { posture, unintentional mouth opening due to hypomimia }\end{array}$ \\
\hline Dysphagia & $\begin{array}{l}\text { Aspiration pneumonia, dementia, depression, } \\
\text { severe motor symptoms }\end{array}$ & $\begin{array}{l}\text { Dysfunction of oral, pharyngeal and esophageal swallowing, } \\
\text { central cholinergic dysfunction } \\
\text { Test: Bed side screening, cough reflex testing, video } \\
\text { fluoroscopy, manometry }\end{array}$ \\
\hline Gastric dysfunction & $\begin{array}{l}\text { Nausea, vomiting, early satiety, postprandial } \\
\text { fullness }\end{array}$ & $\begin{array}{l}\text { Response fluctuation with levodopa } \\
\text { Test: gastric emptying scintigraphy, }{ }^{13} \mathrm{C} \text { sodium octanoate } \\
\text { breath test, electrogastrography }\end{array}$ \\
\hline $\begin{array}{l}\text { H. pylori infection and small } \\
\text { intestinal bacterial overgrowth }\end{array}$ & Motor severity worsening in PD & Serology, urea breath test, serum antigen test \\
\hline $\begin{array}{l}\text { Constipation and defecatory } \\
\text { dysfunction }\end{array}$ & $\begin{array}{l}\text { Infrequent bowel movements, failed defecation } \\
\text { attempts, sense of incomplete rectal emptying } \\
\text { at defecation }\end{array}$ & $\begin{array}{l}\text { Slow transit constipation, dyssynergic defecation. } \\
\text { Test: radioopaque marker study for colonic transit, wireless } \\
\text { motility capsule, anorectal manometry, rectal balloon } \\
\text { expulsion, defecography }\end{array}$ \\
\hline
\end{tabular}

PD, Parkinson's disease; H. pylori, Helicobacter pylori. 
the CNS may possibly be due to the chronic low-grade inflammation in the gut.

\section{Gut Microbiome Dysbiosis}

The most extensively studied example of microbial association of PD is Helicobacter pylori. The prevalence of $H$. pylori is high and causes motor impairments by hindering the absorption of levodopa, a primary drug used for PD management. ${ }^{65}$ Small intestinal bacterial overgrowth (SIBO), a disorder of excessive bacterial growth in the small intestine, has also been associated with PD. SIBO affects almost one quarter of PD patients with motor impairments and its eradication results in the improvement of motor fluctuations. $^{66,67}$

Fecal microbiota collected from 72 patients with PD and agematched controls has shown higher levels of enterobacteriacea and lower counts for prevotellacea. Prevotella helps in breaking down complex carbohydrates to provide short chain fatty acids (SCFAs) along with thiamine and folate which are by products that promote a healthy intestinal environment. ${ }^{68}$ Decrease in prevotella count is linked to decreased production of these important micro nutrients.
Gut microbes like bacillus spp. are known to produce dopamine and gut microbiota accounts for almost half of the dopamine production in the body. ${ }^{69,70}$

Cyanobacteria, present in minute quantities in the GI tract, is believed to produce $\beta$-N-methyl amino-L-alanine (BMAA) which has been found to be increased in the brain of $\mathrm{PD}$, Alzheimer's disease, and amyotrophic lateral sclerosis patients. BMAA is an excitotoxin which activates metabotropic glutamate receptor 5 that causes reduction in the levels of glutathione, a major antioxidant. It is believed that neurons and glial cells cannot control reactive oxygen species (ROS) and reactive nitrogen species (RNS) effectively in the brain. The BMAA protein also has implications over protein misfolding and aggregation particularly in PD, Alzheimer's disease and amyotrophic lateral sclerosis. ${ }^{71}$

\section{Gut Inflammation}

Figure shows an algorithm that represents the gut inflammation driven GI dysfunction mediated PD pathogenesis model. It shows that neurodegeneration in the brain of $\mathrm{PD}$ patients is the ultimate result of a cascade of events that starts from the gut. The initial trig-

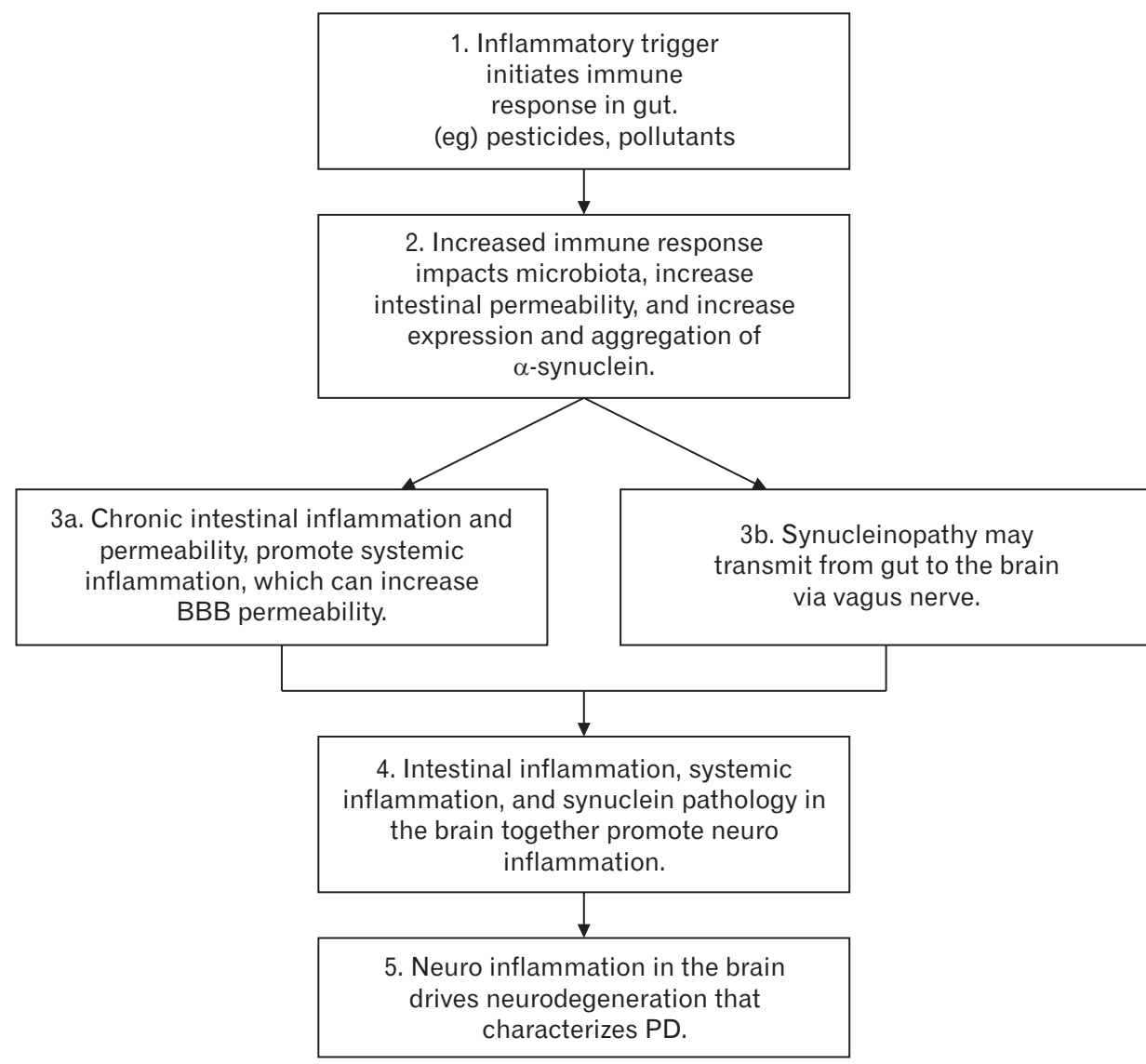

Figure. Gut inflammation driven gastrointestinal dysfunction mediated Parkinson's disease (PD) pathogenesis model. BBB, blood brain barrier. Adapted from Yarandi et $\mathrm{al}^{147}$ and Houser and Tansey. ${ }^{148}$ 
ger caused by the environmental pollutants and pesticides in the gut are crucial. The involvement of gut should therefore be taken into consideration for designing early and reliable diagnostic tools in PD.

\section{Identifying Reliable Early Non-motor Biomarkers}

PD patients exhibit GI dysfunction that precedes almost 2 decades to motor dysfunction. Symptoms like constipation, dysbiosis, altered intestinal permeability and enteric accumulation of $\alpha$-synuclein co-exist with the inflammation in the GI tract. ${ }^{72-75}$ Persistant inflammation in the gut leads to systemic inflammation and ultimately neuroinflammation. Recent studies have reported that gut inflammation and oxidative stress do exist in patients with $\mathrm{PD}^{76,77}$

\section{Constipation}

Constipation is the second most common NMS next to hyposmia in $\mathrm{PD}{ }^{78,79}$ Constipation occurs mainly due to delayed intestinal transit time and its effects are displayed in the small intestine and colon. ${ }^{78,79}$ It is believed that constipation appears as a prodromal symptom years before the motor symptoms appear. ${ }^{80}$ It has been reported that men, in their middle age, with less than one bowel movement per day had 4 times the risk of PD diagnosis for the next 20-25 years compared to men who had normal bowel movements. ${ }^{81}$ Constipated men (bowel movements less than 3 in a week) had 5 times more chance and women with constipation have 3 times more chance of being diagnosed with PD in the next 6 years compared with individuals with normal bowel movement. ${ }^{82}$ Meta-analyses reveal that constipation is more than 2 times common in people who develop PD compared to normal people, ${ }^{83}$ and individuals with constipation have 2 times the chance to have PD within 10 years of their evaluation. ${ }^{84}$ The time duration over which constipation indicates the development of $\mathrm{PD}$ is remarkable. After prospec- tive and retrospective studies, it has been found that constipation becomes apparent at an average of 15.6-24.0 years before PD is diagnosed. ${ }^{33,64,81,85}$ Constipation should, therefore, be considered as one of the earliest and important symptoms of pathological events that lead to PD.

\section{Enteric $\alpha$-Synuclein Deposition}

Alpha synuclein associated enteric abnormality is another intestinal characteristic that has been generally recorded. PD patients have been detected with more frequent and higher levels of intestinal $\alpha$-synuclein compared to the same age matched healthy controls. ${ }^{58,61,62,86-88}$ This has significance as the over-expression of $\alpha$-synuclein causes $\alpha$-synuclein aggregation in the intestine and brain of mice and humans. ${ }^{76,89,90}$ Studies have reported detection of phosphorylated $\alpha$-synuclein in $61.6 \%$ of PD samples and lewy bodies/lewy neutrites in 72.4-100.0\% of PD samples compared to the detection of $0.0-33.0 \%$ of $\alpha$-synuclein in healthy population, ${ }^{91-95}$ suggesting that intestinal synucleinopathy is a relatively sensitive and reliable indicator of PD pathology. Recently it has been reported that distinctive $\alpha$-synuclein immunoreactivity observed in biopsies of intestines of healthy individuals who would develop PD in the later stage, ${ }^{61,62,87}$ give rise to the presumption that abnormal enteric $\alpha$-synuclein appears before neurodegeneration in CNS advances to develop motor symptoms.

\section{Altered Intestinal Barrier Function}

Studies have indicated that patients with PD have increased intestinal permeability compared to healthy controls. ${ }^{76,85,96-98}$ Several studies have also indicated abnormalities in the tight junctions of intestine without complete damage in the mucosa, but characterized by a decrease in the barrier promoting protein levels and disruptions of tight junction networks, ${ }^{76,92,93}$ thus showing a phenotype consistent with low-grade inflammation. ${ }^{72}$ The penetration of $E$. coli into the intestinal mucosa seems to be expressed more frequently in

Table 3. Difference in Fecal Gut Microbiota Composition in Parkinson's Disease Patients Versus Age Matched Control (Adapted From Unger et al ${ }^{103}$ )

\begin{tabular}{lll}
\hline \multicolumn{1}{c}{ Bacterial family } & \multicolumn{1}{c}{ Phylum } & Change in microbial concentration in PD group compared to control \\
\hline Prevotellacea & Bacteroidetes & Decrease \\
Faecalibacterium prausnitzii & Firmicutes & Decrease \\
Lactobacilli/Enterococci & Firmicutes & Decrease \\
Akkarmansia muciniphila & Verrucomicrobia & Increase \\
Bifidobacterium & Actinobacteria & Increase \\
Methanobrevibacter smithii & Archaea & Increase \\
Enterobacteriaceae & Proteobacteria & Increase \\
\hline
\end{tabular}

PD, Parkinson's disease. 
PD patients as compared to controls, and correlate with increased intestinal permeability and oxidative stress and increased enteric $\alpha$-synuclein levels. ${ }^{58}$ Newly diagnosed PD patients have been detected with increased intestinal permeability and this seems to be present since the earliest clinical stages of the disease. ${ }^{58}$

\section{Small Intestinal Bacterial Overgrowth}

The incidence of SIBO, bacterial density, and dysbiosis is greater in the small intestine of $\mathrm{PD}$ patients compared to healthy controls. ${ }^{66,67,99,100}$ The prevalence of SIBO is $25.0-54.5 \%$ in PD patients compared to $8.3-20.0 \%$ in normal controls. The presence of SIBO has been associated with a greater degree of impairment in motor control in PD with fluctuation in levodopa response. ${ }^{67}$

Due to the immediate vicinity of the enteric nervous system (ENS) with fecal products, gut microbiota and its metabolic products are the major candidates that could start the process leading to lewy body formation in the ENS. Two recent studies claim a link between abundance of gut microbiota and PD. ${ }^{68,101}$ According to these studies, bacteria which has the potential to produce SCFAs are reported to be low in PD. SCFAs alter the activity of ENS and increases GI motility. ${ }^{102}$ A shift in gut microbiota may, therefore, have an association with the shift in metabolic products like SCFA. ${ }^{103}$

The difference in fecal gut microbiota composition in $\mathrm{PD}$ patients vs the age matched control has been analysed by real-time quantitative PCR and the data is given in Table 3. The data reveal that bacteria family from phylum bacteroidetes and furmicutes decrease significantly in the fecal samples of PD patients in comparison to age matched controls, whereas the bacteria from phylum verrocomicrobia, actinobacteria, archaea, and proteobacteria increase compared to controls.

The difference in fecal SCFAs of PD patients in comparison to age matched controls has been analysed by gas chromatography and data obtained is given in Table 4 . The data reveal a considerable reduction in the absolute concentrations of acetate, propionate, and butyrate compared to age matched controls thus indicating that

Table 4. Difference in Fecal Short Chain Fatty Acid Composition in Parkinson's Disease Patients Compared to Age Matched Control (Adapted From Unger et $\mathrm{al}^{103}$ )

\begin{tabular}{lc}
\hline \multicolumn{1}{c}{ SCFA } & $\begin{array}{c}\text { Change in SCFA concentration } \\
\text { in PD group compared to control }\end{array}$ \\
\hline Acetate & Decrease \\
Butyrate & Decrease \\
Propionate & Decrease \\
\hline
\end{tabular}

SCFA, short chain fatty acid; PD, Parkinson's disease. gut is the origin of pathological process that causes PD. ${ }^{104}$ Gut microbiota and its metabolic products may therefore be considered as potential factors that could eventually lead to the formation of lewy bodies in the ENS. ${ }^{68,101}$

\section{Gastrointestinal Inflammation}

Prolonged intestinal inflammatory conditions can lead to systemic inflammation and neuroinflammation. The GI inflammation is associated with symptoms like constipation, intestinal permeability, dysbiosis, and higher levels of pathogenic enteric $\alpha$-synuclein (together characterized as GI dysfunction) that appear more than two decades before the onset of motor symptoms. ${ }^{72-75}$ Also, studies have reported that $\mathrm{PD}$ patients show inflammation and oxidative stress in the gut. ${ }^{58,59}$

\section{Current Biomarkers and Investigational Techniques Used}

Individuals with PD have been reported to exhibit increased urinary indoxyl sulfate indicating gut dysbiosis. ${ }^{100}$ Gut microflora is analyzed by the state of the art high-throughput technique. ${ }^{77}$ Denaturing gradient gel electrophoresis and next generation sequencing using high-throughput microbial identification are used to identify different microbial community in PD. Occludins, the tight junction proteins, are important components of tissue barriers and are essential for structural maintenance. ${ }^{105}$ Microbiota changes are linked with alterations in intestinal barrier in GI diseases like irritable bowel syndrome. ${ }^{24}$ Also colonic specimens of PD patients show downregulation of occluding. ${ }^{95}$

Objective measures of PD include leukocyte subset counts, ${ }^{106}$ serum cortisol, $\mathrm{TNF}-\alpha,{ }^{107,108}$ in addition to global motor scores in the peripheral blood mononuclear cell production of cytokines, and nuclear factor kappa-light-chain-enhancer of activated $\mathrm{B}$ cells $(\mathrm{NF}-\kappa \mathrm{B})$ expression. ${ }^{109}$ An increase IL-6 concentration in blood collected four years earlier predicts PD in patients. ${ }^{110}$ Normal concentration of plasma 5-hydroxytryptamine (5-HT) regulates emotion. Gut microbiota may regulate $5-\mathrm{HT}^{111}$ Gut microbiota dysfunction can be linked to decrease in 5-HT and depression in PD patients. ${ }^{112,113}$ Leaky gut, which enables lipopolysaccharide translocation, is involved in inflammation associated with major depression. $^{114}$

Metagenomics technology like proton nuclear magnetic resonance and targeted analysis are used as non-invasive techniques for diagnosing dysbiosis associated with bacteria by profiling biofluids like urine, serum, and plasma. ${ }^{115}$ Selected ion flow tube mass spec- 
trometry is another technique used to detect bacterial dysbiosis by measuring volatile compounds (ethane, methane, pentane, ammonia, dimethyl sulfide) in the breath, stool or urine of PD patients. ${ }^{116}$ A recent study has shown that patients who suffer from depression have increased concentration of isovaleric acid (volatile fatty acid) in their stool. ${ }^{117}$

\section{Correlating Gastrointestinal Mediated Non-motor Symptoms With Established Techniques to Discover Reliable Early Non-invasive Biomarkers}

\section{Correlating Enteric Nervous System Mediated Non-motor Symptoms With Neuro Imaging Techniques}

The NMS in PD include GI symptoms like constipation, enteric $\alpha$-synuclein deposition, altered intestinal barrier function, SIBO, and inflammation in the gut. The presence of these symptoms in the majority of PD patients possibly suggests that these could be considered as preclinical or prodromal biomarkers of PD. It would be more reliable, therefore, if these could be related to the already established investigational techniques.

The established investigational techniques meant for correlation with the NMS of ENS have to be ideal in terms of sensitivity, reproducibility, technical feasiblity, and also cost effective, noninvasive and ultimately they should be thoroughly validated. ${ }^{118}$ Types of the investigational neuroimaging techniques that include single photon emission tomography, positron emission tomography, magnetic resonance imaging, and transcranial sonography (TCS), will give vital clues about the structure and function of the brain in PD patients. These could be used to confirm the extent of accuracy in predicting $\mathrm{PD}$ from early preclinical or prodromal biomarkers of GI origin. ${ }^{119}$

The above-mentioned neuroimaging techniques are noninvasive and can be regularly used to check the integrity of the dopamine system to provide anatomical figures (eg, imbalance in uptake). They give data about the time span of neuronal loss and can be correlated with the disease severity as well. ${ }^{120-123}$

TCS could be used as an ideal imaging technique to correlate with the NMS of the ENS at early stages. TCS can identify echoes of higher density in the midbrain with greater efficiency in hospital as well as community settings. The hyperechogenicity of the midbrain could figure out the increment in the concentration of iron in the substantia nigra of PD patients very early. TCS is thus a promising candidate for discovering early biomarkers. Another state-of-the-art technique called voxel based morphometry that can tell similarities in the structure and differences in the volume, help in identifying PD from other motor disorders in the initial stages of the disease. ${ }^{124,125}$

\section{Correlating Enteric Nervous System Mediated Non-motor Symptoms With Functional or Behavioral Indices}

It is believed that early NMS that reflect degeneration in extranigral regions before the loss of dopamine in the substantia nigra, include altered functions in sleep, olfaction, visuospatial abilities that affect cognition by diminished executive function as well as behavioral change. ${ }^{126}$ The tests which are meant to evaluate these symptoms of $\mathrm{PD}$ are non-invasive, cost effective, easy to perform even at home and/or online by the participants. These functional or behavioral tests include olfaction acuity test (eg, the University of Pennsylvania Smell Identification Test- the UPSIT), RBD screening questionnaire, key board tapping test, the bradykinesia akinesia incoordination test, and accelerometer based examinations. These tests have the potential to correlate with the ENS associated NMS to discover early reliable biomarkers for the consequent improvement of PD screening in various settings. ${ }^{127-129}$

\section{Future Directions for Reliable Early Non-invasive Biomarkers}

Recent studies suggest that biopsies of the GI tract and submandibular gland could be used as potential early biomarkers of PD. These are promising approaches to detect $\alpha$-synuclein neuropathology in the autonomic nervous system of PD cases. ${ }^{130-135}$ In addition, the presence of $\alpha$-synuclein in ENS and submandibular gland is likely to occur at a very early stage in the course of the disease. ${ }^{126,136}$ As of now, the abnormal $\alpha$-synuclein accumulation has been identified in the biopsies within the salivary glands, ${ }^{8,45,56}$ stomach, duodenum, ${ }^{55,57}$ colon and rectum of PD patients. ${ }^{122,130,132,134}$

Analysis of surgical specimens obtained during intervention of diverse pathological conditions demonstrates in vivo $\alpha$-synuclein pathology outside the CNS in PD patients. A recent study has shown the presence of lewy pathology in GI and biliary surgical specimens taken by surgical interventions performed years before the onset of neurological symptoms. This emphasizes the potential utility of surgical specimens for the confirmation of $\alpha$-synuclein pathology for the prediction of PD in patients. ${ }^{127}$

Newer investigational techniques use broader net and more 
global non-targeted strategies, like genomics, proteomics, and metabolomics for identifying multiple biomarkers in tissues from normal and disease affected individuals. ${ }^{137-141}$ Metabolomic evaluations of plasma generated set of metabolites (indicators) are capable of differentiating between PD patients and controls irrespective of medication status. ${ }^{142}$

Recently a study using cerebrospinal fluid and serum of PD affected patients using thorough pathological assessments has shown that these cell free peripheral fluid profiles provide an accurate picture of the cell level pathological events in the deceased tissue of PD patients. ${ }^{143}$ Greater care and attention to standardize clinical assessment and to properly classify different patients, as well as the standardization and proper collection and storage of bio-samples, are vital for these newer approaches to get fruitful results.

There should be a greater focus in future, on studying the current data sets from large population based investigations and also analyzing the archived fluid and tissue samples from large prospective population-based cohort studies. ${ }^{127,144}$ Ultimately the main effort should be made to thoroughly evaluate these results using current bio-informatics and technological approaches.

\section{Conclusion}

Discovering reliable early biomarkers for PD has become the need of the hour for the ailing patients all over the world. Early diagnostic biomarkers are vital for 2 reasons, namely to intervene right at the onset of the disease and to monitor the progress of therapeutic interventions that may slow or stop the course of the disease. Even though loss of dopamine neurons may occur early, clinical motor symptoms of PD like muscular rigidity, body tremors, drooping body posture, difficulty in walking and other symptoms associated with autonomic nervous system show at a later stage in the disease progression, much after the majority of dopamine neurons have degenerated. It is important, therefore, to discover reliable, early, sensitive and specific biomarkers that would enable differential diagnosis, prognosis and effective treatment of PD. Discovery of novel biomarkers no doubt play a major role in the effective treatment of PD with minimum or no side effects. Recent studies have suggested that PD originates in the gut and not in the brain. ${ }^{145}$ It has been shown that a biological link exists between the gut microbiome and $\mathrm{PD}{ }^{146}$ The hypothetical basis for this theory is that the gut bacteria may be responsible for chemicals which are over activating parts of the brain, ${ }^{147}$ leading to $\alpha$-synuclein damage and its deposition which ultimately causes neurodegeneration in the brain resulting in PD ${ }^{148}$ It is important, therefore, to have a forward step to define the specific gut microbes that may contribute to the development of $\mathrm{PD}$, as this could translate into novel biomarkers to identify at-risk patients at a very early stage in the disease progression. These findings may possibly lead to novel therapeutic approaches that avoid the existing complications of drugs delivered to the brain and may also become safer and more effective to the ailing patients.

\section{Financial support: None.}

\section{Conflicts of interest: None.}

Author contributions: Arun T Nair, Shanish Antony, and Gopalakrishnan Ramalingam collected and interpreted data, and drafted the manuscript; and Nanjan M Joghee and Vadivelan Ramachandran was responsible for all aspects of the manuscript.

\section{References}

1. Tysnes OB, Storstein A. Epidemiology of Parkinson's disease. J Neural Transm 2017;124:901-905.

2. Statistics on Parkinson's disease. Parkinson Association of the Carolinas. Available from URL: http://www.parkinsonassociation.org/ (accessed 11 December, 2017).

3. Schapira AH. Recent developments in biomarkers in Parkinson disease. Curr Opin Neurol 2013;26:395-400.

4. Kalia LV, Lang AE. Parkinson's disease. Lancet 2015;386:896-912.

5. Garcia-Ruiz PJ, Chaudhuri KR, Martinez-Martin P. Non-motor symptoms of parkinson's disease a review from the past. J Neurol Sci 2014;338:30-33.

6. Dietert J, Dietert R. The sum of our parts. Scientist 2015;29:44-49.

7. Fond G, Boukouaci W, Chevalier G, et al. The "psychomicrobiotic": targeting microbiota in major psychiatric disorders: a systematic review. Pathol Biol (Paris) 2015;63:35-42.

8. Forsythe P, Sudo N, Dinan T, Taylor VH, Bienenstock J. Mood and gut feelings. Brain Behav Immun 2010;24:9-16.

9. Shakir R. Neurodegenerative noncommunicable diseases (Neurology NCDs). Where are we now? J Neurol Sci 2015;356:1-2.

10. Silberberg D, Anand NP, Michels K, Kalaria RN. Brain and other nervous system disorders across the lifespan - global challenges and opportunities. Nature 2015;527:S151-S154.

11. Wolters ECh, Braak H. Parkinson's disease: premotor clinico-pathological correlations. J Neural Transm Suppl 2006;70:309-319.

12. Sulzer D. Multiple hit hypotheses for dopamine neuron loss in Parkinson's disease. Trends Neurosci 2007;30:244-250.

13. Doudet D, Ruth T. Imaging the brain in Parkinson's disease: BC Med 2001;3:148-152.

14. Rascol O, Payoux P, Ory F, Ferreira JJ, Brefel-Courbon C, Montastruc JL. Limitations of current Parkinson's disease therapy. Ann Neuro 2003;53:S3-12; discussion S12-S15. 
15. Murman DL. Early treatment of Parkinson's disease: opportunities for managed care. Am J Manag Care 2012;18:S183-S188.

16. Lancet Neurology. Building on 50 years of levodopa therapy. Lancet Neurol 2016;15:1.

17. Picillo M, Moccia M, Spina E, Barone P, Pellecchia MT. Biomarkers of Parkinson's disease: recent insights, current challenges, and future prospects. J Parkinsonism Restless Legs Syndr 2016;6:1-13.

18. DeKosky ST, Marek K. Looking backward to move forward: early detection of neurodegenerative disorders. Science 2003;302:830-834.

19. Scherzer C. Interview-Searching for biomarkers in Parkinson's disease. Biomark Med 2009;3:1134.

20. O'Sullivan SS, Williams DR, Gallagher DA, Massey LA, SilveiraMoriyama L, Lees AJ. Nonmotor symptoms as presenting complaints in Parkinson's disease: a clinico pathological study. Mov Disord 2008;23:101-106.

21. Barber TR, Klein JC, Mackay CE, Hu MTM. Neuroimaging in premotor Parkinson's disease. Neuroimage Clin 2017;15:215-227.

22. Cryan JF, O'Mahony SM. The microbiome-gut-brain axis: from bowel to behavior. Neurogastroenterol Motil 2011;23:187-192.

23. De Palma G, Collins SM, Bercik P, Verdu EF. The microbiota-gutbrain axis in gastrointestinal disorders: stressed bugs, stressed brain or both? J Physiol 2014;592:2989-2997.

24. Mayer EA, Tillisch K, Bradesi S. Review article: modulation of the brain-gut axis as a therapeutic approach in gastrointestinal disease. Aliment Pharmacol Ther 2006;24:919-933.

25. Cryan JF, Dinan TG. Mind-altering microorganisms: the impact of the gut microbiota on brain and behaviour. Nat Rev Neurosci 2012;13:701712.

26. Aziz Q, Doré J, Emmanuel A, Guarner F, Quigley EM. Gut microbiota and gastrointestinal health: current concepts and future directions. Neurogastroenterol Motil 2013;25:4-15.

27. Bonaz BL, Bernstein CN. Brain-gut interactions in inflammatory bowel disease. Gastroenterology 2013;144:36-49.

28. Davari S, Talaei SA, Alaei H, Salami M. Probiotics treatment improves diabetes-induced impairment of synaptic activity and cognitive function: behavioral and electrophysiological proofs for microbiome-gut-brain axis. Neuroscience 2013;240:287-296.

29. Grenham S, Clarke G, Cryan JF, Dinan TG. Brain-gut-microbe communication in health and disease. Front Physiol 2011;2:94.

30. Mayer EA. Gut feelings: the emerging biology of gut-brain communication. Nat Rev Neurosci 2011;12:453-466.

31. Quigley EM, Monsour HP. The gut microbiota and nonalcoholic fatty liver disease. Semin Liver Dis 2015;35:262-269.

32. O'Mahony SM, Hyland NP, Dinan TG, Cryan JF. Maternal separation as a model of brain-gut axis dysfunction. Psychopharmacology (Berl) 2011;214:71-88.

33. Rhee SH, Pothoulakis C, Mayer EA. Principles and clinical implications of the brain-gut-enteric microbiota axis. Nat Rev Gastroenterol Hepatol 2009;6:306-314.

34. Thakur AK, Shakya A, Husain GM, Emerald M, Kumar V. Gut-microbiota and mental health: current and future perspectives. J Pharmacol Clin Toxicol 2014;2:1016.
35. Blekhman R, Goodrich JK, Huang K, et al. Host genetic variation impacts microbiome composition across human body sites. Genome Biol 2015;16:191.

36. Bäckhed F, Roswall J, Peng Y, et al. Dynamics and stabilization of the human gut microbiome during the first year of life. Cell Host Microbe 2015;17:690-703.

37. Debnath M, Venkatasubramanian G, Berk M. Fetal programming of schizophrenia: select mechanisms. Neurosci Biobehav Rev 2015;49:90104.

38. Dominguez-Bello MG, Blaser MJ. Asthma: undoing millions of years of coevolution in early life? Sci Transl Med 2015;7:307fs39.

39. Van Opstal EJ, Bordenstein SR. Rethinking heritability of the microbiome. Science 2015;349:1172-1173.

40. Dominguez-Bello MG, De Jesus-Laboy KM, Shen N, et al. Partial restoration of the microbiota of cesarean-born infants via vaginal microbial transfer. Nat Med 2016;22:250-253.

41. Gomez de Agüero M, Ganal-Vonarburg SC, Fuhrer T, et al. The maternal microbiota drives early postnatal innate immune development. Science 2016;351:1296-1302

42. Al-Asmakh M, Hedin L. Microbiota and the control of blood-tissue barriers. Tissue Barriers 2015;3:e1039691.

43. McKenney PT, Pamer EG. From hype to hope: the gut microbiota in enteric infectious disease. Cell 2015;163:1326-1332.

44. Scharschmidt TC, Vasquez KS, Truong HA, et al. A wave of regulatory $\mathrm{T}$ cells into neonatal skin mediates tolerance to commensal microbes. Immunity 2015;43:1011-1021.

45. Wang Z, Roberts AB, Buffa JA, et al. Non-lethal inhibition of gut microbial tri methylamine production for the treatment of atherosclerosis. Cell 2015;163:1585-1595.

46. Yang I, Corwin EJ, Brennan PA, Jordan S, Murphy JR, Dunlop A. The infant microbiome: Implications for infant health and neurocognitive development. Nurs Res 2016;65:76-88.

47. Hinde K, Lewis ZT. Mother's littlest helpers. Science 2015;348:14271428 .

48. Meadow JF, Altrichter AE, Bateman AC, et al. Humans differ in their personal microbial cloud. Peer J 2015;3:e1258.

49. Zeevi D, Korem T, Zmora N, et al. Personalized nutrition by prediction of glycemic responses. Cell 2015;163:1079-1094.

50. Zuker CS. Food for the brain. Cell 2015;161:9-11.

51. Collins SM, Surette M, Bercik P. The interplay between the intestinal microbiota and the brain. Nat Rev Microbiol 2012;10:735-742.

52. Mayer EA, Tillisch K, Gupta A. Gut/brain axis and the microbiota. J Clin Invest 2015;125:926-938.

53. Sampson TR, Mazmanian SK. Control of brain development, function, and behavior by the microbiome. Cell Host Microbe 2015;17:565-576.

54. Friedland RP. Mechanisms of molecular mimicry involving the microbiota in neurodegeneration. J Alzheimers Dis 2015;45:349-362.

55. Del Tredici K, Rüb U, De Vos RA, Bohl JR, Braak H. Where does Parkinson disease pathology begin in the brain? J Neuropathol Exp Neurol 2002;61:413-426.

56. Ueki A, Otsuka M. Life style risks of Parkinson's disease: association between decreased water intake and constipation. J Neurol 
2004;251(suppl 7):vII18-vII23.

57. Cersosimo MG, Benarroch EE. Pathological correlates of gastrointestinal dysfunction in Parkinson's disease. Neurobiol Dis 2012;46:559-564.

58. Forsyth CB, Shannon KM, Kordower JH, et al. Increased intestinal permeability correlates with sigmoid mucosa alpha-synuclein staining and endotoxin exposure markers in early parkinson's disease. PLoS One 2011;6:e28032.

59. Devos D, Lebouvier T, Lardeux B, et al. Colonic inflammation in parkinson's disease. Neurobiol Dis 2013;50:42-48.

60. Poewe W. Non-motor symptoms in Parkinson's disease. Eur J Neurol 2008;15(suppl 1):14-20.

61. Braak H, de Vos RA, Bohl J, Del Tredici K. Gastric alpha-synuclein immunoreactive inclusions in meissner's and auerbach's plexuses in cases staged for parkinson's disease related brain pathology. Neurosci Lett 2006;396:67-72.

62. Shannon KM, Keshavarzian A, Dodiya HB, Jakate S, Kordower JH. Is alpha-synuclein in the colon a biomarker for premotor Parkinson's disease? Evidence from 3 cases. Mov Disord 2012;27:716-719.

63. Kieburtz K, Wunderle KB. Parkinson's disease: evidence for environmental risk factors. Mov Disord: 2013;28:8-13.

64. Savica R, Carlin JM, Grossardt BR, et al. Medical records documentation of constipation preceding Parkinson disease: a case-control study. Neurology 2009;73:1752-1758.

65. Çamci G, Oğuz S. Association between Parkinson's disease and helicobacter pylori. J Clin Neurol 2016;12:47-50.

66. Fasano A, Bove F, Gabrielli M, et al. The role of small intestinal bacterial overgrowth in Parkinson's disease. Mov Disord 2013;28:1241-1249.

67. Tan AH, Mahadeva S, Thalha AM, et al. Small intestinal bacterial overgrowth in Parkinson's disease. Parkinsonism Relat Disord 2014;20:535540.

68. Keshavarzian A, Green SJ, Engen PA, et al. Colonic bacterial composition in Parkinson's disease. Mov Disord 2015;1351-1360.

69. Eisenhofer G, Aneman A, Friberg P, et al. Substantial production of dopamine in the human gastrointestinal tract. J Clin Endocrinol Metab 1997;82:3864-3871.

70. Wall R, Cryan JF, Ross RP, Fitzgerald GF, Dinan TG, Stanton C. Bacterial neuroactive compounds produced by psychobiotics. Adv Exp Med Biol 2014;817:221-239.

71. Brenner SR. Blue-green algae or cyanobacteria in the intestinal microflora may produce neurotoxins such as Beta-N-Methylamino-L-Alanine (BMAA) which may be related to development of Amyotrophic lateral sclerosis, Alzheimer's disease and Parkinson-dementia-complex in humans and equine motor neuron disease in horses. Med Hypotheses 2013;80:103.

72. Edelblum KL, Turner JR. The tight junction in inflammatory disease: communication breakdown. Curr Opin Pharmacol 2009;9:715-720.

73. Scher JU, UbedaC, Artacho A, et al. Decreased bacterial diversity characterizes the altered gut microbiota in patients with psoriatic arthritis, resembling dysbiosis in inflammatory bowel disease. Arthritis Rheumatol 2015;67:128-139.

74. Rana SV. Small intestinal bacterial overgrowth and orocecal transit time in patients of inflammatory bowel disease. Dig Dis Sci 2013;58:2594-
2598.

75. Kelly LP, Carvey PM, Keshavarzian A, et al. Progression of intestinal permeability changes and alphasynuclein expression in a mouse model of Parkinson's disease. Mov Disord 2014;29:999-1009.

76. Klein C, Westenberger A. Genetics of Parkinson's disease. Cold Spring Harb Perspect Med 2012;2:a008888.

77. Fraher MH, O’Toole PW, Quigley EM. Techniques used to characterize the gut microbiota : a guide for the clinician. Nat Rev Gastroenterol Hepatol 2012;9:312-322

78. Dutkiewicz J, Szlufik S, Nieciecki M, et al. Small intestine dysfunction in Parkinson's disease. J Neural Transm (Vienna) 2015;122:1659-1661.

79. Sakakibara R, Odaka T, Uchiyama T, et al. Colonic transit time and rectoanal video manometry in Parkinson's disease. J Neurol Neurosurg Psychiatry 2003;74:268-272.

80. Goldman JG, Postuma R. Premotor and non-motor features of Parkinson's disease. Curr Opin Neurol 2014;27:434-441.

81. Abbott RD, Petrovitch H, White LR, et al. Frequency of bowel movements and the future risk of Parkinson's disease. Neurology 2001;57:456-462.

82. Gao X, Chen H, Schwarzschild MA, Ascherio A. A prospective study of bowel movement frequency and risk of Parkinson's disease. Am J Epidemiol 2011;174:546-551.

83. Chen H, Zhao EJ, Zhang W, et al. Meta-analyses on prevalence of selected Parkinson's non motor symptoms before and after diagnosis. Transl Neurodegener 2015;4:1.

84. Adams-Carr KL, Bestwick JP, Shribman S, Lees A, Schrag A, Noyce AJ. Constipation preceding Parkinson's disease: a systematic review and meta-analysis. J Neurol Neurosurg Psychiatry 2015;87:710-716.

85. Postuma RB, Gagnon JF, Pelletier A, Montplaisir J. Prodromal autonomic symptoms and signs in parkinson's disease and dementia with lewy bodies. Mov Disord 2013;28:597-604.

86. Gold A, Turkalp ZT, Munoz DG. Enteric alpha-synuclein expression is increased in Parkinson's disease but not Alzheimer's disease. Mov Disord 2013;28:237-240.

87. Hilton D, Stephens M, Kirk L, et al. Accumulation of alpha-synuclein in the bowel of patients in the pre-clinical phase of Parkinson's disease. Acta Neuropathol 2014;127:235-241.

88. Shannon KM, Keshavarzian A, Mutlu E, et al. Alpha-synuclein in colonic submucosa in early untreated parkinson's disease. Mov Disord 2012;27:709-715.

89. Wang L, Fleming SM, Chesselet MF, Taché Y. Abnormal colonic motility in mice over expressing human wild-type alpha-synuclein. Neuroreport 2008;19:873-876.

90. Hallett PJ, McLean JR, Kartunen A, Langston JW, IsacsonO. Alphasynuclein over expressing transgenic mice shows internal organ pathology and autonomic deficits. Neurobiol Dis 2012;47:258-267.

91. Beach TG, Adler CH, Sue LI, et al. Multi-organ distribution of phosphorylated alpha-synuclein histopathology in subjects with lewy body disorders. Acta Neuropathol 2010;119:689-702.

92. Lebouvier T, Neunlist M, Bruley des Varannes S, et al. Colonic biopsies to assess the neuropathology of parkinson's disease and its relationship with symptoms. PLoS One 2010;5:e12728. 
93. Wakabayashi K, Takahashi H, Takeda S, Ohama E, Ikuta F. Parkinson's disease: the presence of lewy bodies in auerbach's and meissner's plexuses. Acta Neuropathol 1988;76:217-221.

94. Lebouvier T, Chaumette T, Damier P, et al. Pathological lesions in colonic biopsies during parkinson's disease. Gut 2008;57:1741-1743.

95. Clairembault T, Leclair-Visonneau L, Coron E, et al. Structural alterations of the intestinal epithelial barrier in parkinson's disease. Acta Neuropathol Commun 2015;3:12.

96. Shaikh M, Rajan K, Forsyth CB, Voigt RM, Keshavarzian A. Simultaneous gas-chromatographic urinary measurement of sugar probes to assess intestinal permeability: use of time course analysis to optimize its use to assess regional gut permeability. Clin Chim Acta 2015;442:24-32.

97. Hasegawa S, Goto S, Tsuji H, et al. Intestinal dysbiosis and lowered serum lipopolysaccharide-binding protein in parkinson's disease. PLoS One 2015;10:e0142164.

98. Salat-Foix D, Tran K, Ranawaya R, Meddings J, Suchowersky O. Increased intestinal permeability and Parkinson disease patients: chicken or egg? Can J Neurol Sci 2012;39:185-188.

99. Gabrielli M, Bonazzi P, Scarpellini E, et al. Prevalence of small intestinal bacterial overgrowth in parkinson's disease. Mov Disord 2011;26:889892.

100. Cassani E, Barichella M, Dancello R, et al. Increased urinary indoxyl sulfate (indican): new insights into gut dysbiosis in Parkinson's disease. Parkinsonism Relat Disord 2015;21:389-393.

101. Scheperjans F, Aho V, Pereira PA, et al. Gut microbiota are related to Parkinson's disease and clinical phenotype. Mov Disord 2015;30:350358.

102. Soret R, Chevalier J, De Coppet P, et al. Short-chain fatty acids regulate the enteric neurons and control gastrointestinal motility in rats. Gastroenterology 2010;138:1772-1782.

103. Unger MM, Spiegel J, Dillmann KU, et al. Short chain fatty acids and gut microbiota differ between patients with Parkinson's disease and agematched controls. Parkinsonism Relat Disord 2016;32:66-72.

104. Mukherjee A, Biswas A, Das SK. Gut dysfunction in Parkinson's disease. World J Gastroenterol 2016;22:5742-5752.

105. Förster C. Tight junctions and the modulation of barrier function in disease. Histochem Cell Biol 2008;130:55-70.

106. Dobbs RJ, Charlett A, Dobbs SM, et al. Leukocyte-subset counts in idiopathic Parkinsonism provide clues to a pathogenic pathway involving small intestinal bacterial over growth. A surveillance study. Gut Pathog 2012;4:12.

107. Charlett A, Dobbs RJ, Purkiss AG, et al. Cortisol is higher in Parkinsonism and associated with gait deficit. Acta Neurol Scand 1998;97:7785.

108. Dobbs RJ, Charlett A, Purkiss AG, Dobbs SM, Weller C, Peterson DW. Association of circulating TNF-alpha and IL-6 with ageing and Parkinsonism. Acta Neurol Scand 1999;100:34-41.

109. Reale M, Iarlori C, Thomas A, et al. Peripheral cytokines profile in parkinson's disease. Brain Behav Immun 2009;23:55-63.

110. Chen H, O'Reilly EJ, Schwarzschild MA, Ascherio A. Peripheral inflammatory biomarkers and risk of Parkinson's disease. Am J Epidemiol 2008;167:90-95.
111. Yano JM, Yu K, Donaldson GP, et al. Indigenous bacteria from the gut microbiota regulates host serotonin biosynthesis. Cell 2015;161:264-276.

112. Hsu YT, Liao CC, Chang SN, et al. Increased risk of depression in patients with Parkinson disease: a nationwide cohort study. Am J Geriatr Psychiatry 2015;23:934-940.

113. Tong Q, Zhang L, Yuan Y, et al. Reduced plasma serotonin and 5-hydroxyindoleacetic acid levels in Parkinson's disease are associated with nonmotor symptoms. Parkinsonism Relat Disord 2015;21:882-887.

114. Maes M, Kubera M, Leunis JC. The gut-brain barrier in major depression: Intestinal mucosal dysfunction with an increased translocation of LPS from gram negative enterobacteria (leaky gut) plays a role in the inflammatory pathophysiology of depression. Neuro Endocrinol Lett 2008;29:117-124.

115. Schicho R, Shaykhutdinov R, Ngo J, et al. Quantitative metabolomic profiling of serum, plasma, and urine by H NMR spectroscopy discriminates between patients with inflammatory bowel disease and healthy individuals. J Proteome Res 2012;11:3344-3357.

116. Van Berkel JJ, Dallinga JW, Möller GM, et al. A profile of volatile organic compounds in breath discriminates COPD patients from controls. Respir Med 2010;104:557-563.

117. Szczesniak O, Hestad KA, Hanssen JF, Rudi K. Isovaleric acid in stool correlates with human depression. Nutr Neurosci 2016;19:279-283.

118. Liepelt-Scarfone I, Gauss K Maetzler W, et al. Evaluation of progression markers in the premotor phase of Parkinson's disease: the progression markers in the premotor phase study. Neuroepidemiology 2013;41:174-182.

119. Saeed U, Compagnone J, Aviv RI, et al. Imaging biomarkers in Parkinson's disease and Parkinsonian symptoms: current and emerging concepts. Transl Neurodegener 2017;6:8.

120. Sharma S, Moon CS. Biomarkers in Parkinson's disease (recent update). Neurochem Int 2013;63:201-229.

121. Berg D, Godau J, Walter U. Transcranial sonography in movement disorders. Lancet Neurol 2008;7:1044-1055.

122. Gaig C, Tolosa E. When does Parkinson's disease begin? Mov Disord 2009;24(suppl 2):S656-S664.

123. Tuite PJ, Mangia S. Magnetic resonance imaging (MRI) in Parkinson's disease. J Alzheimers Dis Parkinsonism 2013;(suppl 1):001.

124. Poewe W, Mahlknecht P. Combined assessment of midbrain hyperechogenicity, hyposmia and motor asymmetry improves diagnostic accuracy in early Parkinson's disease. Expert Rev Neurother 2012;12:911-914.

125. Shao N, Yang J, Li J, Shang HF. Voxelwise meta-analysis of gray matter anomalies in progressive supranuclear palsy and parkinson's disease using anatomic likelihood estimation. Front Hum Neurosci 2014;8:63.

126. Berg D, Lang AE, Postuma RB, et al. Change the research criteria for the diagnosis of Parkinson's disease: obstacles and opportunities. Lancet Neurol 2013;12:514-524.

127. Berg D, Marek K, Ross GW, Poewe W. Defining at-risk populations for Parkinson 's disease: lessons from ongoing studies. Mov Disord 2012;27:656-665.

128. Lerche S, Hobert M, Brochmann K, et al. Mild parkinsonian signs in the elderly - Is there an association with PD? Crossectional findings in 992 individuals. PLoS One 2014;9:e92878. 
129. Noyce AJ, Bestwick JP, Silveira-Moriyam L et al. PREDICT-PD: Identifying risk of Parkinson's disease in the community: methods and baseline results. J Neurol Neurosur Psychiatry 2014;85:31-37.

130. Berg D, Postuma RB, Bloem B, et al. Time to redefine PD? Introductory statement of the MDS task force on the definition of Parkinson's disease. Mov Disord 2014;29:454-462.

131. Bhidayasiri R, Reichmann H. Different diagnostic criteria for Parkinson disease: what are the pitfalls? J Neural Transm (Vienna) 2013;120:619625.

132. Lees AJ, Hardy J, Revesz T. Parkinson's disease. Lancet 2009; 373:2055-2066.

133. Deutch AY. Parkinson's disease redefined. Lancet Neurol 2013;12:422423.

134. Wang N, Gibbons CH, Lafo J, Freeman R. $\alpha$-synuclein in cutaneous autonomic nerves. Neurol 2013;81:1604-1610.

135. del Campo M, Mollenhauer B, Bertolotto A. Recommendations to standardize preanalytical confounding factors in Alzheimer's and Parkinson's disease cerebrospinal fluid biomarkers: an update. Biomark Med 2012;6:419-430.

136. Van Dijk KD, Teunissen CE, Drukarch B, et al. Diagnostic cerebrospinal fluid biomarkers for Parkinson's disease: a pathogenetically based approach. Neurobiol Dis 2010;39:229-241.

137. Mielke MM, Maetzler W. A 'bird's eye' view on the current status and potential benefits of blood biomarkers for Parkinson's disease. Biomarkers Med 2014;8:225-227.

138. Saracchi E, Fermi S, Brighina L. Emerging candidate biomarkers for Parkinson's disease: a review. Aging Dis 2013;5:27-34.

139. Rappaport SM. Biomarkers intersect with the exposome. Biomarkers
2012;17:483-489.

140. Bogdanov M, Matson WR, Wang L, et al. Metabolomic profiling to develop blood biomarkers for Parkinson's disease. Brain 2008;131(pt 2):389-396.

141. Rappaport SM, Barupal DK, Wishart D, Vineis P, Scalbert A. The blood exposome and its role in discovering causes of disease. Environ Health Perspect 2014;122:769-774.

142. Ahmed SS, Santosh W, Kumar S, Christlet HTT. Metabolic profiling of Parkinson's disease: evidence of biomarker from gene expression analysis and rapid neural network detection. J Biomed Sci 2009;16:63.

143. Burgos K, Malenica I, Metpally R, et al. Profiles of extracellular miRNA in cerebrospinal fluid and serum from patients with Alzheimer's and Parkinson's diseases correlate with disease status and features of pathology. PLoS One 2014;9:e94839.

144. DeKosky ST, Gandy S. Environmental exposures and the risk for Alzheimer disease: can we identify the smoking guns? JAMA Neurol 2014;71:273-275.

145. Mulak A, Bonaz B. Brain-gut-microbiota axis in Parkinson's disease. World J Gastroenterol 2015;21:10609-10620.

146. Principi N, Esposito S. Gut microbiota and central nervous system development. J Infect 2016;73:536-546.

147. Yarandi SS, Peterson DA, Treisman GJ, Moran TH, Pasricha PJ. Modulatory effects of gut microbiota on the central nervous system: how gut could play a role in neuropsychiatric health and diseases. J Neurogastroenterol Motil 2016;22:201-212.

148. Houser MC, Tansey MG. The gut-brain axis: is intestinal inflammation a silent driver of Parkinson's disease pathogenesis? NPJ Parkinson's Dis $2017 ; 3: 3$. 